\title{
The Role of the Central (Pulkovo) Observatory in Southern Hemisphere Star Observations
}

\author{
V.K. Abalakin, A.A. Arkharov, Y.N. Gnedin, I.I. Kanayev, D.D. \\ Polojentsev and H.I. Potter
}

Pulkovo Observatory, Saint-Petersburg, 196140, Russia

The Nicholas Central Astronomical Observatory in Pulkovo, near SaintPetersburg, was inaugurated as Russia's principal astronomical institution on 19 (7) August 1839. Construction of the observatory had begun in 1834 following the edict of the Russian Emperor Nicholas I, who had invited as his adviser Professor Friedrich Georg Wilhelm Struve, the Director of the Dorpat Observatory. Emperor Nicholas I appointed Wilhelm Struve as the Director of the new observatory. With regard to funding, the emperor had given Struve a carte blanche to enable him to order from German telescope makers the best astrometric instruments designed according to Struve's own ideas. Hence Wilhelm Struve is to be considered as the co-founder of Pulkovo Observatory.

The main scientific goals of the new observatory consisted in the determination of precise star positions, compilation of catalogues of star positions and proper motions, and derivation of numerical values of astronomical constants and also geodetic parameters. Over the next decades, the famous Pulkovo star catalogues were compiled on the basis of high precision star observations; they were assigned weights exceeding by a factor of 3.6 those allotted to the Greenwich catalogues. Thus, by the end of the 19th century, the American astronomer Benjamin Althorp Gould had called Pulkovo Observatory the "Astronomical Metropole of the World".

In 1958, at the Xth IAU General Assembly in Moscow, Professor M. Zverev from Russia (then: USSR) and F. Rutllant from Chile agreed on participation of Pulkovo astronomers in astrometric observations of stars in the southern hemisphere using the instruments of Cerero Calan Observatory of the University of Santiago de Chile. Pulkovo astronomers made observations in Chile between 1962 and 1972, having installed at the new observation site at Sierra El Roblo several Pulkovo telescopes. The Pulkovo astronomers compiled and published five star catalogues derived from meridian observations made jointly with Chilean colleagues. These catalogues were included in the southern section of the FK5 fundamental catalogue (Fricke et al. 1991), the results of joint work between Russian and Chilean astronomers having been published in 15 papers.

In 1983 a new astrometric expeditionary team was sent from Pulkovo Observatory to Bolivia. Here a new Russo-Bolivian observing station (now known as the Santa Anna National Observatory of Bolivia) was established. The site was near the town of Tarija in Southern Bolivia at an altitude of 2000 metres. The observational campaign was successfully continued up to 1990 , and a new photographic astrometric catalogue of positions of southern hemisphere stars was compiled. Known as the PHOCAT-S Catalogue, this formed a basis for the southern part of the PPM Catalogue (Bastian \& Röser 1993) compiled and 
published by colleagues from the Astronomisches Rechen-Institut at Heidelberg. Accordingly, 39 papers were published making use of astrometric and astrophysical observations made in Tarija (Bolivia), jointly by Russian, Ukrainian and Bolivian astronomers.

\section{References}

Fricke, W., Schwan, H., Corbin, T., Bastian, U., Bien, R., Cole, C., Jackson, E., Jährling, R., Jahreiß, H., Lederle, T., \& Röser, S. 1991, "Fifth Fundamental Catalogue (FK5) Part II. The FK5 Extension - New Fundamental Stars", (Karlsruhe: Veröffentlichungen Astronomisches Rechen-Institut Heidelberg, No.33)

Bastian, U., \& Röser, S. 1993, "PPM Star Catalogue", Vols III and IV (Heidelberg: Spektrum Akademischer Verlag) 